\title{
THE INVERSE TAYLOR OPERATOR AND COMPLETELY MONOTONIC FUNCTIONS
}

\section{DEMETRIOS P. KANOUSSIS}

Kalamos Attikis

19017

Greece

e-mail: dkanoussis@gmail.com

\begin{abstract}
A new expansion of a function, inspired by the Taylor series expansion, is introduced, and various examples of functions admiting such new expansion, are given. In addition, it is shown that the new expansion, when applied to functions being completely monotonic, leads to some quite interesting results and applications. Finally, a new approach is suggested towards obtaining the solution of the difference equation $\Delta y(x)=g(x)$, and examples involving the gamma and psi functions are given.
\end{abstract}

\section{Introduction}

Let $g(x)$ be a real valued, continuous function, defined on the interval $(\alpha, \infty)$. Three classical operators which can apply to $g(x)$, are the derivative operator

$$
D=\frac{d}{d x}
$$

2010 Mathematics Subject Classification: 39A60, 33B15.

Keywords and phrases: difference operators, difference equations, complete monotonicity, gamma function, digamma (or psi) function.

Received January 10, 2017

(C) 2017 Scientific Advances Publishers 
the shift operator

$$
E(g(x))=g(x+1)
$$

and the difference operator

$$
\Delta(g(x))=g(x+1)-g(x)=(E-I)(g(x)),
$$

where $I$ is the identity operator.

Higher order operators are defined similarly,

$$
\begin{gathered}
D^{\kappa}(g(x))=\frac{d^{\kappa}(g(x))}{d x^{\kappa}}=g^{(\kappa)}(x), \\
E^{\kappa}(g(x))=g(x+\kappa), \\
\Delta^{\kappa}(g(x))=(E-I)^{\kappa}(g(x))=\sum_{j=0}^{\kappa}(-1)^{\kappa-j}\left(\begin{array}{l}
\kappa \\
j
\end{array}\right) E^{j}(g(x))=\sum_{j=0}^{\kappa}(-1)^{\kappa-j}\left(\left.\right|_{j} ^{\kappa}\right)_{j}(x+j),
\end{gathered}
$$

where $\kappa$ is any positive integer.

It is easily shown that if $\kappa$ and $\lambda$ are any two positive integers, the operators $\Delta^{\kappa}$ and $D^{\lambda}$ commute

$$
\Delta^{\kappa}\left(D^{\lambda}(g(x))\right)=D^{\lambda}\left(\Delta^{\kappa}(g(x))\right)
$$

provided that $g(x)$ is $\lambda$ times differentiable on $(\alpha, \infty)$.

Let us further assume that $g(x)$, within its interval of definition, admits an infinite Taylor series expansion, of the form

$$
g(x+1)=g(x)+\frac{g^{(1)}(x)}{1 !}+\frac{g^{(2)}(x)}{2 !}+\frac{g^{(3)}(x)}{3 !}+\cdots=\sum_{\kappa=0}^{\infty} \frac{D^{\kappa}(g(x))}{\kappa !}
$$

or equivalently,

$$
\Delta(g(x))=\left(e^{D}-I\right)(g(x)),
$$


from which one, formally, obtains the operator equivalency,

$$
\Delta=e^{D}-I, \quad \text { or } \quad D=\ln (I+\Delta)
$$

The operator $e^{D}$ could be called the Taylor operator, in which case, an appropriate name for $\ln (I+\Delta)$, would be the inverse Taylor operator.

However, it is not clear what is the meaning of the operator $\ln (I+\Delta)$. One possible way to interpret $\ln (I+\Delta)$, is to consider the expansion

$$
\ln (I+\Delta)=\Delta-\frac{\Delta^{2}}{2}+\frac{\Delta^{3}}{3}-\frac{\Delta^{4}}{4}+\cdots=\sum_{n=1}^{\infty} \frac{(-1)^{n-1}}{n} \Delta^{n},
$$

in accordance to the well-known expansion for $\ln (1+x),-1<x \leq 1$.

In this context, (1.9) leads to

$$
D(g(x))=\left(\Delta-\frac{\Delta^{2}}{2}+\frac{\Delta^{3}}{3}-\frac{\Delta^{4}}{4}+\cdots\right)(g(x)) .
$$

It is worth mentioning that (1.11) was obtained quite formally, and therefore there is no a priori warranty about its general validity. On the contrary, (1.11) should motivate an investigation towards the direction of finding functions $g(x)$ for which (1.11) is valid.

Simple, preliminary calculations show that such functions do exist. For example, if $g(x)=e^{-x}$ or if $g(x)$ is any polynomial of $x,(1.11)$ is valid (this is shown in the next section). On the other hand, if $g(x)=e^{x}$, (1.11) is not valid, since the infinite series in the right side of (1.11) does not converge.

In the sequel of this paper, we will show that the class of functions $g(x)$, satisfying (1.11) is considerably rich. Expanding a function $g(x)$, or rather the derivative $D(g(x))$, according to (1.11), usually reveals new properties of the function at hand, and often leads to some quite interesting results and unexpected applications ([9], [10]). 
Definition 1. We say that a real valued function $g(x)$, belongs to the class $K_{\alpha}$, if

$$
D(g(x))=\left(\Delta-\frac{\Delta^{2}}{2}+\frac{\Delta^{3}}{3}-\frac{\Delta^{4}}{4}+\cdots\right)(g(x))=\sum_{\kappa=1}^{\infty} \frac{(-1)^{\kappa-1}}{\kappa} \Delta^{\kappa}(g(x)),
$$

for all $x \in(\alpha, \infty)$.

In particular $K_{-\infty}$ will represent the class of functions for which expansion (1.12) is valid for all real values of $x(-\infty<x<\infty)$, while $K_{0}$ will represent the class of functions satisfying (1.12) for all positive real values of $x(0<x<\infty)$.

Proving various theorems, in the rest of this paper, we shall make use of the following elementary identities:

(i) $\Delta(f(x) g(x))=\Delta(f(x)) g(x)+f(x+1) \Delta(g(x))$.

(ii) If $f(x)=x,(\Delta(f(x))=\Delta(x)=1),(1.13)$ reduces to

$$
\Delta(x g(x))=g(x)+(x+1) \Delta(g(x)) .
$$

(iii) Taking successive differences of (1.14), one may easily show that

$$
\Delta^{\kappa}(x g(x))=\kappa \Delta^{\kappa-1}(g(x))+(x+\kappa) \Delta^{\kappa}(g(x)), \text { where } \kappa=1,2,3, \ldots
$$

(iv) It is well-known that the $\ln (1-x)$ function, admits the following power series expansion:

$$
-\ln (1-x)=\sum_{\kappa=1}^{\infty} \frac{x^{\kappa}}{\kappa}, \quad-1 \leq x<1
$$

Since $0 \leq 1-e^{-t}<1$, for all $t \in[0, \infty)$, (1.16) leads to

$$
\sum_{\kappa=1}^{\infty} \frac{\left(1-e^{-t}\right)^{\kappa}}{\kappa}=t, \quad 0 \leq t<\infty
$$




\section{Various Functions Belonging to Some Class $K_{\alpha}$}

In this section, we shall list a number of functions satisfying (1.12), for appropriate values of the parameter $\alpha$.

Theorem 1. All monomials $x^{n}, n \in \mathbb{N}$, are in $K_{-\infty}$.

Proof. We shall use induction on $n$, to show that $x^{n} \in K_{-\infty}$, for all $n \in \mathbb{N}$. For $n=1$, obviously $D(x)=\Delta(x)=1$.

Let us now assume that $x^{n} \in K_{-\infty}$, for some $n \in \mathbb{N}$, meaning that

$$
D\left(x^{n}\right)=\sum_{\kappa=1}^{\infty} \frac{(-1)^{\kappa-1}}{\kappa} \Delta^{\kappa}\left(x^{n}\right)=\sum_{\kappa=1}^{n} \frac{(-1)^{\kappa-1}}{\kappa} \Delta^{\kappa}\left(x^{n}\right),
$$

since $\Delta^{\kappa}\left(x^{n}\right)=0$ for $\kappa=n+1, n+2, n+3, \ldots$

We shall show that $x^{n+1} \in K_{-\infty}$, as well.

Making use of (1.15), with $g(x)=x^{n}$, one obtains

$$
\Delta^{\kappa}\left(x^{n+1}\right)=\kappa \Delta^{\kappa-1}\left(x^{n}\right)+(x+\kappa) \Delta^{\kappa}\left(x^{n}\right), \quad \kappa=1,2,3, \ldots, n,
$$

while

$$
\Delta^{n+1}\left(x^{n+1}\right)=(n+1) \Delta^{n}\left(x^{n}\right)+(x+n+1) \Delta^{n+1}\left(x^{n}\right)=(n+1) \Delta^{n}\left(x^{n}\right),
$$

since $\Delta^{n+1}\left(x^{n}\right)=0$.

In that sense the infinite series in (1.12) terminates, the last term

being $\frac{(-1)^{n}}{n+1} \Delta^{n+1}\left(x^{n+1}\right)$. Using (2.1), (2.2), and (2.3), one may easily show that

$$
\begin{aligned}
\sum_{\kappa=1}^{n+1} \frac{(-1)^{\kappa-1}}{\kappa} \Delta^{\kappa}\left(x^{n+1}\right) & =x^{n}+x \sum_{\kappa=1}^{n} \frac{(-1)^{\kappa-1}}{\kappa} \Delta^{\kappa}\left(x^{n}\right) \\
& =x^{n}+x D\left(x^{n}\right)=(n+1) x^{n}=D\left(x^{n+1}\right),
\end{aligned}
$$

and this completes the proof. 
An immediate consequence of Theorem 1, is the following.

Theorem 2. All polynomials are in $K_{-\infty}$.

Theorem 3. The function $g(x)=e^{-x} \in K_{-\infty}$.

Proof. The $\kappa$-th difference of $g(x)=e^{-x}$, is easily found to be

$$
\Delta^{\kappa}\left(e^{-x}\right)=(-1)^{\kappa}\left(1-e^{-1}\right)^{\kappa} e^{-x}, \quad \kappa \in \mathbb{N} .
$$

Making use of (2.4) and (1.17) (applied at $t=1$ ), the infinite series

$$
\sum_{\kappa=1}^{\infty} \frac{(-1)^{\kappa-1}}{\kappa} \Delta^{\kappa}\left(e^{-x}\right)=(-1) e^{-x} \sum_{\kappa=1}^{\infty} \frac{\left(1-e^{-1}\right)^{\kappa}}{\kappa}=(-1) e^{-x}=D\left(e^{-x}\right),
$$

and the proof is completed.

Theorem 4. The trigonometric functions $\cos x$ and $\sin x$ are in $K_{-\infty}$.

Proof. Let us consider the function $w(x)=e^{i x}, x \in \mathbb{R}$.

The $\kappa$-th difference of $w(x)$, is easily found to be

$$
\Delta^{\kappa}\left(e^{i x}\right)=(-1)^{\kappa}\left(1-e^{i}\right)^{\kappa} e^{i x}, \quad \kappa=1,2,3, \ldots
$$

Since $\left|1-e^{i}\right|=|(1-\cos 1)-i \sin 1|=2 \sin \left(\frac{1}{2}\right)<1$,

$$
\begin{aligned}
\sum_{\kappa=1}^{\infty} \frac{(-1)^{\kappa-1}}{\kappa} \Delta^{\kappa}\left(e^{i x}\right) & =(-1) e^{i x} \sum_{\kappa=1}^{\infty} \frac{\left(1-e^{i}\right)^{\kappa}}{\kappa} \\
& =(-1)^{2} e^{i x} \ln \left(1-1+e^{i}\right)=i e^{i x}=-\sin x+i \cos x,
\end{aligned}
$$

and equating real and imaginary parts, yields

$$
\sum_{\kappa=1}^{\infty} \frac{(-1)^{\kappa-1}}{\kappa} \Delta^{\kappa}(\cos x)=-\sin x=D(\cos x)
$$


and

$$
\sum_{\kappa=1}^{\infty} \frac{(-1)^{\kappa-1}}{\kappa} \Delta^{\kappa}(\sin x)=\cos x=D(\sin x)
$$

which completes the proof.

Theorem 5. If $p$ is any positive real number, then $x^{-p} \in K_{0}$.

Proof. Starting with the definition of the gamma function,

$$
\Gamma(p)=\int_{0}^{\infty} e^{-u} u^{p-1} d u, \quad p>0
$$

and making the substitution $u=t x,(x>0)$, one obtains

$$
x^{-p}=\frac{1}{\Gamma(p)} \int_{0}^{\infty} e^{-x t} t^{p-1} d t, \quad x>0, \quad p>0,
$$

from which

$$
\Delta^{\kappa}\left(x^{-p}\right)=\frac{(-1)^{\kappa}}{\Gamma(p)} \int_{0}^{\infty} e^{-x t}\left(1-e^{-t}\right)^{\kappa} t^{p-1} d t, \quad \kappa \in \mathbb{N} .
$$

The infinite series

$$
\sum_{\kappa=1}^{\infty} \frac{(-1)^{\kappa-1}}{\kappa} \Delta^{\kappa}\left(x^{-p}\right)=\frac{(-1)}{\Gamma(p)} \sum_{\kappa=1}^{\infty} \int_{0}^{\infty} \frac{e^{-x t}\left(1-e^{-t}\right)^{\kappa} t^{p-1}}{\kappa} d t
$$

and since the integrand is positive for all $\kappa$ and $t$, interchange of summation with integration is permissible, by Tonelli's theorem, therefore,

$$
\begin{aligned}
\sum_{\kappa=1}^{\infty} \frac{(-1)^{\kappa-1}}{\kappa} \Delta^{\kappa}\left(x^{-p}\right) & =\frac{(-1)}{\Gamma(p)} \int_{0}^{\infty} e^{-x t}\left(\sum_{\kappa=1}^{\infty} \frac{\left(1-e^{-t}\right)^{\kappa}}{\kappa}\right) t^{p-1} d t \\
& =\frac{(-1)}{\Gamma(p)} \int_{0}^{\infty} e^{-x t} t^{p} d t
\end{aligned}
$$


(by virtue of (1.17)), or

$$
\sum_{\kappa=1}^{\infty} \frac{(-1)^{\kappa-1}}{\kappa} \Delta^{\kappa}\left(x^{-p}\right)=\frac{(-1)}{\Gamma(p)} x^{-(p+1)} \Gamma(p+1),
$$

(from (2.7)), and since

$$
\Gamma(p+1)=p \Gamma(p)
$$

one finally obtains

$$
\sum_{\kappa=1}^{\infty} \frac{(-1)^{\kappa-1}}{\kappa} \Delta^{\kappa}\left(x^{-p}\right)=-p x^{-(p+1)}=D\left(x^{-p}\right)
$$

and the proof is completed.

We have thus far shown that the class $K_{\alpha}$ is not empty. Many important functions belong to some $K_{\alpha}$.

In the next section, we shall show that, under certain additional conditions, one may significantly extend the class of functions already belonging to some $K_{\alpha}$ (see Theorems 12,13, 14, and 15).

\section{Completely Monotonic Functions}

Definition 2. A function $g(x)$ is said to be completely monotonic on $(\alpha, \infty)$, if

(i) $g(x)$ possesses derivatives of all orders, and

(ii) $(-1)^{\kappa} D^{\kappa}(g(x))>0$ for $\kappa=0,1,2, \ldots$.

Conditions (i) and (ii) imply that for each $\kappa$, the function $(-1)^{\kappa} D^{\kappa}(g(x))$, within its interval of definition, is positive, decreasing and concave upwards. 
For example, the functions $e^{-x}$ and $x^{-p}(p>0)$, are completely monotonic on $(0, \infty)$. Also, if $g(x)$ is the Laplace transform of a positive function $P(t), 0 \leq t<\infty$, i.e., if $g(x)=\int_{0}^{\infty} e^{-x t} P(t) d t, g(x)$ is completely monotonic on $(0, \infty)$ (see [1], [5], [14]).

If $g(x)$ is completely monotonic on $(\alpha, \infty)$ and $G_{n}(x)$ is any $n$-th antiderivative of $g(x)$, then for every $x \in(\alpha, \infty)$,

$$
(-1)^{\kappa} D^{\kappa+n}\left(G_{n}(x)\right)>0, \quad \kappa=0,1,2, \ldots
$$

Functions of complete monotonicity have attracted special attention by various researchers, (see, for example, [2], [4], [6], and [7]).

In this section, we shall derive some fundamental properties of completely monotonic functions, belonging to some class $K_{\alpha}$, which eventually lead to quite interesting results and applications.

Theorem 6. If $g(x)$ is completely monotonic on $(\alpha, \infty)$, then for each $n=0,1,2, \ldots$, the function

$$
(-1)^{n} \Delta^{n}(g(x))
$$

is also completely monotonic within the same interval.

In particular, $(-1)^{n} \Delta^{n}(g(x))$ is positive and decreasing for all $n$.

Proof. For $n=0,(-1)^{n} \Delta^{n}(g(x))=g(x)$ is completely monotonic, by assumption.

Also, since $(-1)^{\kappa} D^{\kappa}(g(x))>0, \kappa=0,1,2, \ldots$, the function $(-1)^{\kappa} D^{\kappa}(g(x))$, is for each $\kappa$, positive and decreasing, meaning that

$$
\Delta\left((-1)^{\kappa} D^{\kappa}(g(x))\right)<0, \text { or }(-1)^{\kappa} D^{\kappa}((-1) \Delta(g(x)))>0,
$$

(by virtue of (1.6)), which in turn proves the validity of Theorem 6 , for $n=1$. 
Similarly, since $(-1) \Delta(g(x))$ is completely monotonic, as shown in (3.2),

$$
\Delta\left((-1)^{\kappa} D^{\kappa}((-1) \Delta(g(x)))\right)<0, \text { or }(-1)^{\kappa} D^{\kappa}\left((-1)^{2} \Delta^{2}(g(x))\right)>0,
$$

which proves Theorem 6 , for $n=2$.

In a quite similar manner, proceeding step by step, we can prove Theorem 6, for all values of $n$.

So, in general,

$$
(-1)^{\kappa} D^{\kappa}\left((-1)^{n} \Delta^{n}(g(x))\right)>0,
$$

which for $\kappa=0$, implies that $(-1)^{n} \Delta^{n}(g(x))>0$, while for $\kappa=1$ implies that $(-1)^{n} \Delta^{n}(g(x))$ is decreasing.

Theorem 7. Let $g(x)$ be a given function. If

(i) $g(x) \in K_{\alpha}$, and

(ii) $g(x)$ is completely monotonic on $(\alpha, \infty)$,

then the infinite series

$$
\sum_{\kappa=1}^{\infty} \frac{(-1)^{\kappa-1}}{\kappa} \Delta^{\kappa}(g(x)),
$$

converges absolutely and uniformly on $\alpha<x_{0} \leq x<\infty$.

Proof. Since $g(x) \in K_{\alpha}$, series (3.4), at $x=x_{0}$ converges to $\left.D(g(x))\right|_{x=x_{0}}=D\left(g\left(x_{0}\right)\right)$. Also, due to condition (ii), on $\alpha<x_{0} \leq x<\infty$, we have, by virtue of Theorem 6 ,

$$
0<(-1)^{\kappa} \Delta^{\kappa}(g(x)) \leq\left.(-1)^{\kappa} \Delta^{\kappa}(g(x))\right|_{x=x_{0}}=(-1)^{\kappa} \Delta^{\kappa}\left(g\left(x_{0}\right)\right),
$$

for $\kappa=1,2,3, \ldots$ 
If we now consider the absolute values of the terms in series (3.4), we have

$$
0<\left|\frac{(-1)^{\kappa-1}}{\kappa} \Delta^{\kappa}(g(x))\right|=\frac{(-1)^{\kappa}}{\kappa} \Delta^{\kappa}(g(x)) \leq \frac{(-1)^{\kappa}}{\kappa} \Delta^{\kappa}\left(g\left(x_{0}\right)\right),
$$

(from (3.5)), and since the series

$$
\sum_{\kappa=1}^{\infty} \frac{(-1)^{\kappa}}{\kappa} \Delta^{\kappa}\left(g\left(x_{0}\right)=(-1) \sum_{\kappa=1}^{\infty} \frac{(-1)^{\kappa-1}}{\kappa} \Delta^{\kappa}\left(g\left(x_{0}\right)\right),\right.
$$

converges to $(-1) D\left(g\left(x_{0}\right)\right)>0$, (because $\left.g(x) \in K_{\alpha}\right)$ application of Weierstrass M-Test, shows that series (3.4) is absolutely and uniformly convergent on $\alpha<x_{0} \leq x<\infty$, and the proof is completed.

Theorem 8. If $g(x)$ is a function completely monotonic on $(\alpha, \infty)$, and $G_{m}(x)$ is any $m$-th antiderivative of $g(x),\left(G_{0}(x)=g(x)\right)$, then

$$
(-1)^{\kappa} \Delta^{\kappa+m}\left(G_{m}(x)\right)>0
$$

where $\kappa=0,1,2, \ldots$

Proof. For $m=0$, (3.6) is obvious, since $G_{0}(x)=g(x)$.

Let $m=1$. The function $g(x)=D\left(G_{1}(x)\right)$ is completely monotonic, by assumption, therefore,

$$
(-1)^{\kappa} D^{\kappa}\left(D G_{1}(x)\right)>0,
$$

which by virtue of the Theorem 6 , implies

$$
(-1)^{\kappa} \Delta^{\kappa}\left(D G_{1}(x)\right)>0, \text { or } D\left((-1)^{\kappa} \Delta^{\kappa}\left(G_{1}(x)\right)\right)>0
$$

which in turn means that the function $(-1)^{\kappa} \Delta^{\kappa}\left(G_{1}(x)\right)$ is increasing, within $(\alpha, \infty)$, i.e.,

$$
\Delta\left((-1)^{\kappa} \Delta^{\kappa}\left(G_{1}(x)\right)\right)>0 \text {, or }(-1)^{\kappa} \Delta^{\kappa+1}\left(G_{1}(x)\right)>0,
$$

and this proves Theorem 8, for $m=1$. 
Also, since $G_{1}(x)=D\left(G_{2}(x)\right)$, we have

$$
(-1)^{\kappa} \Delta^{\kappa+1}\left(D G_{2}(x)\right)>0, \text { or } D\left((-1)^{\kappa} \Delta^{\kappa+1}\left(G_{2}(x)\right)\right)>0,
$$

meaning that the function $(-1)^{\kappa} \Delta^{\kappa+1}\left(G_{2}(x)\right)$ is increasing, i.e.,

$$
\Delta\left((-1)^{\kappa} \Delta^{\kappa+1}\left(G_{2}(x)\right)\right)>0, \text { or }(-1)^{\kappa} \Delta^{\kappa+2}\left(G_{2}(x)\right)>0,
$$

and this completes the proof for $m=2$.

Proceeding similarly, one can prove Theorem 8 , for $m=3,4,5, \ldots$.

For brievety and economy, in the sequel of this paper, we shall make use of the simplified notation $\Delta^{\kappa} G_{m}(x)$ or $\Delta^{\kappa} G_{m}$ and $D^{\kappa} G_{m}(x)$ or $D^{\kappa} G_{m}$ instead of $\Delta^{\kappa}\left(G_{m}(x)\right)$ and $D^{\kappa}\left(G_{m}(x)\right)$, respectively.

Theorem 9. Let $g(x)$ be a given function and $G_{m}(x)$ be any $m$-th antiderivative of $g(x)$. If

(i) $g(x) \in K_{\alpha}$, and

(ii) $g(x)$ is completely monotonic on $(\alpha, \infty)$,

then the infinite series

$$
\sum_{\kappa=1}^{\infty} \frac{(-1)^{\kappa-1}}{\kappa} \Delta^{\kappa} G_{m}(x),
$$

converges absolutely and uniformly on $\alpha<x_{0} \leq x<\infty$.

Proof. We shall prove Theorem 9, gradually, step by step.

We start with $m=1$, in which case $G_{1}(x)$ is the first antiderivative of $g(x)$. Since, by assumption $g(x)$ is completely monotonic on $(\alpha, \infty)$, for each $\kappa=0,1,2, \ldots$ the function $(-1)^{\kappa} D^{\kappa} g(x)$ is positive and decreasing, i.e., 


$$
0<(-1)^{\kappa} D^{\kappa} g(x+1)<\int_{x}^{x+1}(-1)^{\kappa}\left(D^{\kappa} g(t)\right) d t<(-1)^{\kappa} D^{\kappa} g(x),
$$

or

$$
0<(-1)^{\kappa} D^{\kappa} g(x+1)<(-1)^{\kappa} D^{\kappa}\left(\Delta G_{1}(x)\right)<(-1)^{\kappa} D^{\kappa} g(x)
$$

or

$$
(-1)^{\kappa} D^{\kappa}\left(g-\Delta G_{1}\right)>0 .
$$

In deriving (3.8), we have used the fact that

$$
\begin{aligned}
\int_{x}^{x+1}\left(D^{\kappa} g(t)\right) d t & =D^{\kappa}\left(\int_{x}^{x+1} g(t) d t\right) \\
& =D^{\kappa}\left(G_{1}(x+1)-G_{1}(x)\right)=D^{\kappa}\left(\Delta G_{1}(x)\right) .
\end{aligned}
$$

Making use of Theorem 6, (3.8) implies

$$
(-1)^{\kappa} \Delta^{\kappa}\left(g-\Delta G_{1}\right)>0, \text { or }(-1)^{\kappa} \Delta^{\kappa+1} G_{1}<(-1)^{\kappa} \Delta^{\kappa} g, \kappa=0,1,2, \ldots,
$$

which when combined with (3.6) (with $m=1$ ), finally yields

$$
0<\frac{(-1)^{\kappa}}{\kappa+1} \Delta^{\kappa+1} G_{1}(x)<\frac{(-1)^{\kappa}}{\kappa+1} \Delta^{\kappa} g(x)<\frac{(-1)^{\kappa}}{\kappa} \Delta^{\kappa} g(x) \leq \frac{(-1)^{\kappa}}{\kappa} \Delta^{\kappa} g\left(x_{0}\right)
$$

for $\kappa=1,2,3, \ldots$, and $\alpha<x_{0} \leq x<\infty$.

The last inequality in (3.10) stems directly from (3.5).

The series

$$
\sum_{\kappa=1}^{\infty} \frac{(-1)^{\kappa}}{\kappa} \Delta^{\kappa} g\left(x_{0}\right)=(-1) \sum_{\kappa=1}^{\infty} \frac{(-1)^{\kappa-1}}{\kappa} \Delta^{\kappa} g\left(x_{0}\right)=(-1) D g\left(x_{0}\right)>0,
$$

since by assumption $g(x) \in K_{\alpha}$, therefore application of Weierstrass M-Test, shows that the series

$$
\sum_{\kappa=1}^{\infty} \frac{(-1)^{\kappa}}{\kappa+1} \Delta^{\kappa+1} G_{1}=-\frac{1}{2} \Delta^{2} G_{1}+\frac{1}{3} \Delta^{3} G_{1}-\frac{1}{4} \Delta^{4} G_{1}+\cdots,
$$


is absolutely and uniformly convergent on $\alpha<x_{0} \leq x<\infty$, and therefore,

$$
\begin{aligned}
\sum_{\kappa=0}^{\infty} \frac{(-1)^{\kappa}}{\kappa+1} \Delta^{\kappa+1} G_{1} & =\sum_{\kappa=1}^{\infty} \frac{(-1)^{\kappa-1}}{\kappa} \Delta^{\kappa} G_{1} \\
& =\Delta G_{1}-\frac{1}{2} \Delta^{2} G_{1}+\frac{1}{3} \Delta^{3} G_{1}-\frac{1}{4} \Delta^{4} G_{1}+\cdots,
\end{aligned}
$$

is also absolutely and uniformly convergent on $\alpha<x_{0} \leq x<\infty$, and this completes the proof for $m=1$.

We next prove the theorem for $m=2$, in which case $G_{2}(x)$ is a second antiderivative of $g(x)$.

Since $(-1)^{\kappa} D^{\kappa}\left(D G_{1}\right)>0$, by assumption (ii), for each $\kappa=0,1,2, \ldots$, the function $(-1)^{\kappa} D^{\kappa+1} G_{1}$ is positive and decreasing, meaning that

$$
0<(-1)^{\kappa} D^{\kappa+1} G_{1}(x+1)<\int_{x}^{x+1}(-1)^{\kappa}\left(D^{\kappa+1} G_{1}(t)\right) d t<(-1)^{\kappa} D^{\kappa+1} G_{1}(x)
$$

or

$$
0<(-1)^{\kappa} D^{\kappa+1} G_{1}(x+1)<(-1)^{\kappa} D^{\kappa+1}\left(\Delta G_{2}(x)\right)<(-1)^{\kappa} D^{\kappa+1} G_{1}(x),
$$

or

$$
(-1)^{\kappa+1} D^{\kappa+1}\left(\Delta G_{2}-G_{1}\right)>0 .
$$

By virtue of Theorem 6, (3.11) implies

$$
(-1)^{\kappa+1} \Delta^{\kappa+1}\left(\Delta G_{2}-G_{1}\right)>0 \text {, or }(-1)^{\kappa} \Delta^{\kappa+2} G_{2}<(-1)^{\kappa} \Delta^{\kappa+1} G_{1},
$$

or taking into consideration (3.6) with $m=2$,

$$
0<(-1)^{\kappa} \Delta^{\kappa+2} G_{2}<(-1)^{\kappa} \Delta^{\kappa+1} G_{1}, \quad \kappa=0,1,2, \ldots,
$$


or even more,

$$
\begin{aligned}
0<\frac{(-1)^{\kappa}}{\kappa+2} \Delta^{\kappa+2} G_{2} & <\frac{(-1)^{\kappa}}{\kappa+2} \Delta^{\kappa+1} G_{1}<\frac{(-1)^{\kappa}}{\kappa+1} \Delta^{\kappa+1} G_{1} \\
& <\frac{(-1)^{\kappa}}{\kappa} \Delta^{\kappa}(g(x)) \leq \frac{(-1)^{\kappa}}{\kappa} \Delta^{\kappa}\left(g\left(x_{0}\right)\right),
\end{aligned}
$$

for $\kappa=1,2,3, \ldots$, and $\alpha<x_{0} \leq x<\infty$.

The last inequality in (3.13) stems directly from (3.10).

Since, by assumption (i),

$$
\sum_{\kappa=1}^{\infty} \frac{(-1)^{\kappa}}{\kappa} \Delta^{\kappa} g\left(x_{0}\right)=(-1) \sum_{\kappa=1}^{\infty} \frac{(-1)^{\kappa-1}}{\kappa} \Delta^{\kappa} g\left(x_{0}\right)=(-1) D g\left(x_{0}\right)>0,
$$

application again of Weierstrass M-Test shows that the series

$$
\sum_{\kappa=1}^{\infty} \frac{(-1)^{\kappa}}{\kappa+2} \Delta^{\kappa+2} G_{2}=-\frac{1}{3} \Delta^{3} G_{2}+\frac{1}{4} \Delta^{4} G_{2}-\frac{1}{5} \Delta^{5} G_{2}+\cdots,
$$

is absolutely and uniformly convergent on $\alpha<x_{0} \leq x<\infty$, and therefore the series

$$
\sum_{\kappa=1}^{\infty} \frac{(-1)^{\kappa-1}}{\kappa} \Delta^{\kappa} G_{2}=\Delta G_{2}-\frac{1}{2} \Delta^{2} G_{2}+\frac{1}{3} \Delta^{3} G_{2}-\frac{1}{4} \Delta^{4} G_{2}+\cdots,
$$

is also absolutely and uniformly convergent on $\alpha<x_{0} \leq x<\infty$, and this completes the proof for $m=2$.

Proceeding along similar lines, one can prove (3.7) gradually, for $m=3,4,5, \ldots$.

Theorem 10. If $g(x)$ is completely monotonic on $(\alpha, \infty)$, such that

$$
\lim _{x \rightarrow \infty} g(x)=0
$$


then

(i)

$$
\lim _{x \rightarrow \infty} g^{\prime}(x)=0, \text { and }
$$

(ii)

$$
\lim _{x \rightarrow \infty} \Delta^{\kappa} G_{m}(x)=0, \text { for } \kappa \geq m
$$

where $G_{m}(x)$ is any $m$-th antiderivative of $g(x)$.

Proof. (i) Since, by assumption $g(x) \rightarrow 0$ as $x \rightarrow \infty$, the

$$
\lim _{x \rightarrow \infty}(\Delta g(x))=\lim _{x \rightarrow \infty}(g(x+1)-g(x))=0-0=0 .
$$

Also, since the function $(-1) \operatorname{Dg}(x)$ is positive and decreasing, on $(\alpha, \infty)$

$$
0<(-1) D g(x+1)<\int_{x}^{x+1}(-1)(D g(t)) d t<(-1) D g(x)
$$

or

$$
0<(-1) D g(x+1)<(-1) \Delta g(x)<(-1) D g(x)
$$

i.e.,

$$
\Delta g(x)<g^{\prime}(x+1)<0, x \in(\alpha, \infty),
$$

and since $\lim _{x \rightarrow \infty}(\Delta g(x))=0$, as proved, we have

$$
\lim _{x \rightarrow \infty} g^{\prime}(x+1)=\lim _{x \rightarrow \infty} g^{\prime}(x)=0,
$$

and the proof is completed.

(ii) Let $G_{1}(x)$ be the first antiderivative of $g(x)$. From (3.9), applied at $\kappa=0$, and Theorem 8 , with $m=1$, we have

$$
0<\Delta G_{1}(x)<g(x)
$$

and since $\lim _{x \rightarrow \infty} g(x)=0$, we infer that $\lim _{x \rightarrow \infty} \Delta G_{1}(x)=0$. Given that, if the first difference of a function tends to zero, as $x \rightarrow \infty$, then all 
higher order differences of the function tend to zero, as well, as $x \rightarrow \infty$, we conclude that

$$
\Delta^{2} G_{1}(x), \Delta^{3} G_{1}(x), \Delta^{4} G_{1}(x), \ldots
$$

tend to zero, as $x \rightarrow \infty$, and this proves (3.15), for $m=1$.

Let now $G_{2}(x)$ be a second antiderivative of $g(x)$. From (3.12), applied at $\kappa=0$, and Theorem 8 , with $m=2$, we have

$$
0<\Delta^{2} G_{2}(x)<\Delta G_{1}(x)
$$

and since $\lim _{x \rightarrow \infty} \Delta G_{1}(x)=0$, as proved already, we infer that $\Delta^{2} G_{2}(x) \rightarrow 0$ as $x \rightarrow \infty$, and therefore all higher order differences, i.e.,

$$
\Delta^{3} G_{2}(x), \Delta^{4} G_{2}(x), \Delta^{5} G_{2}(x), \ldots
$$

tend to zero, as $x \rightarrow \infty$, and this proves (3.15), for $m=2$.

In a similar manner (3.15) is proved, gradually, for $m=3,4,5, \ldots$.

Theorem 11. Let $g(x)$ be a given function, and $G_{m}(x)$ be any $m$-th antiderivative of $g(x)$. If

(i) $g(x) \in K_{\alpha}$,

(ii) $g(x)$ is completely monotonic on $(\alpha, \infty)$, and

(iii) $\lim _{x \rightarrow \infty} g(x)=0$,

then, if we define

$$
\begin{aligned}
R_{m}(x)= & \frac{1}{m} \Delta^{m} G_{m}(x)-\frac{1}{m+1} \Delta^{m+1} G_{m}(x) \\
& +\frac{1}{m+2} \Delta^{m+2} G_{m}(x)-\frac{1}{m+3} \Delta^{m+3} G_{m}(x)+\cdots,
\end{aligned}
$$

then

$$
\lim _{x \rightarrow \infty} R_{m}(x)=0
$$


Proof. Let us define the quantity,

$$
Q_{m}(x)=-\frac{1}{m+1} \Delta^{m+1} G_{m}(x)+\frac{1}{m+2} \Delta^{m+2} G_{m}(x)-\frac{1}{m+3} \Delta^{m+3} G_{m}(x)+\cdots .
$$

Then,

$$
R_{m}(x)=\frac{1}{m} \Delta^{m} G_{m}(x)+Q_{m}(x) .
$$

The function $Q_{m}(x)$ satisfies the following inequality:

$$
0<Q_{m}(x)<(-1) \operatorname{Dg}(x)
$$

which can be deduced easily from (3.10), (3.13) and their obvious generalizations, i.e.,

$$
0<\frac{(-1)^{\kappa}}{\kappa+m} \Delta^{\kappa+m} G_{m}(x)<\frac{(-1)^{\kappa}}{\kappa} \Delta^{\kappa} g(x), \quad m=1,2,3, \ldots,
$$

and the fact that

$$
\sum_{\kappa=1}^{\infty} \frac{(-1)^{\kappa}}{\kappa} \Delta^{\kappa} g(x)=(-1) \sum_{\kappa=1}^{\infty} \frac{(-1)^{\kappa-1}}{\kappa} \Delta^{\kappa} g(x)=(-1) D g(x),
$$

since by assumption, $g(x) \in K_{\alpha}$.

The

$$
\lim _{x \rightarrow \infty} \operatorname{Dg}(x)=0,
$$

by Theorem 10 (i), therefore (3.19) implies that

$$
\lim _{x \rightarrow \infty} Q_{m}(x)=0
$$

Also since

$$
\lim _{x \rightarrow \infty} \Delta^{m} G_{m}(x)=0
$$

by Theorem 10 (ii), (3.18) finally yields

$$
\lim _{x \rightarrow \infty} R_{m}(x)=\frac{1}{m} \lim _{x \rightarrow \infty} \Delta^{m} G_{m}(x)+\lim _{x \rightarrow \infty} Q_{m}(x)=0,
$$

which completes the proof of Theorem 11 . 
Theorem 12. Let $g(x)$ be a given function, and $G_{m}(x)$ be any $m$-th antiderivative of $g(x)$. If

(i) $g(x) \in K_{\alpha}$,

(ii) $g(x)$ is completely monotonic on $(\alpha, \infty)$, and

(iii) $\lim _{x \rightarrow \infty} g(x)=0$,

then

$$
G_{m}(x) \in K_{\alpha}
$$

Proof. We shall prove Theorem 12, gradually, step by step, starting with $m=1$, in which case $G_{1}(x)$ represents the first antiderivative of $g(x)$.

By virtue of Theorem 9, the series

$$
S_{1}(x)=\sum_{\kappa=1}^{\infty} \frac{(-1)^{\kappa-1}}{\kappa} \Delta^{\kappa} G_{1}(x)=R_{1}(x)
$$

where $R_{1}(x)$ is obtained from (3.16) with $m=1$, converges absolutely and uniformly on $\alpha<x_{0} \leq x<\infty$, while the series of the derivatives of the terms in (3.21), i.e., the series

$$
\left.\sum_{\kappa=1}^{\infty} D\left(\frac{(-1)^{\kappa-1}}{\kappa} \Delta^{\kappa} G_{1}(x)\right)=\sum_{\kappa=1}^{\infty} \frac{(-1)^{\kappa-1}}{\kappa} \Delta^{\kappa}\left(D G_{1}(x)\right)=\sum_{\kappa=1}^{\infty} \frac{(-1)^{\kappa-1}}{\kappa} \Delta^{\kappa} g(x)\right)
$$

by Theorem 7, also converges absolutely and uniformly over the same interval.

Application of the well-known theorem, about the term by term differentiation of a uniformly convergent series, yields

$$
\begin{aligned}
D S_{1}(x) & =D\left(\sum_{\kappa=1}^{\infty} \frac{(-1)^{\kappa-1}}{\kappa} \Delta^{\kappa} G_{1}(x)\right)=\sum_{\kappa=1}^{\infty} \frac{(-1)^{\kappa-1}}{\kappa} D\left(\Delta^{\kappa} G_{1}(x)\right) \\
& =\sum_{\kappa=1}^{\infty} \frac{(-1)^{\kappa-1}}{\kappa} \Delta^{\kappa}\left(D G_{1}(x)\right)=\sum_{\kappa=1}^{\infty} \frac{(-1)^{\kappa-1}}{\kappa} \Delta^{\kappa} g(x)=D g(x),
\end{aligned}
$$


since, by assumption $g(x) \in K_{\alpha}$, or equivalently, and taking (3.21) into account,

$$
D S_{1}(x)=D g(x), \text { or } S_{1}(x)=R_{1}(x)=g(x)+c_{1},
$$

where, up to this point, $c_{1}$ is an arbitrary constant. Taking the limit of both sides in (3.23), as $x \rightarrow \infty$, and noting that $\lim _{x \rightarrow \infty} R_{1}(x)=0$, (by Theorem 11), and that $\lim _{x \rightarrow \infty} g(x)=0$ (by assumption), $c_{1}=0$, and finally

$$
S_{1}(x)=\sum_{\kappa=1}^{\infty} \frac{(-1)^{\kappa-1}}{\kappa} \Delta^{\kappa} G_{1}(x)=g(x)=D G_{1}(x)
$$

meaning that $G_{1}(x) \in K_{\alpha}$, and this completes the proof of Theorem 12, for $m=1$.

Let now $G_{2}(x)$ be the second antiderivative of $g(x)$, or the same, the first antiderivative of $G_{1}(x)$. By virtue of Theorem 9 , the series

$$
S_{2}(x)=\sum_{\kappa=1}^{\infty} \frac{(-1)^{\kappa-1}}{\kappa} \Delta^{\kappa} G_{2}(x)=\Delta G_{2}(x)-R_{2}(x)
$$

where $R_{2}(x)$ is obtained from (3.16) with $m=2$, converges absolutely and uniformly, on $\alpha<x_{0} \leq x<\infty$, while the series of the derivatives of the terms in (3.24), i.e.,

$$
\sum_{\kappa=1}^{\infty} D\left(\frac{(-1)^{\kappa-1}}{\kappa} \Delta^{\kappa} G_{2}(x)\right)=\sum_{\kappa=1}^{\infty} \frac{(-1)^{\kappa-1}}{\kappa} \Delta^{\kappa}\left(D G_{2}(x)\right)=\sum_{\kappa=1}^{\infty} \frac{(-1)^{\kappa-1}}{\kappa} \Delta^{\kappa} G_{1}(x)
$$

again by Theorem 9, converges absolutely and uniformly, over the same interval, to the function $D G_{1}(x)$, since $G_{1}(x) \in K_{\alpha}$, as proved already. 
We can therefore differentiate term by term the series in (3.24), to obtain

$$
D S_{2}(x)=\sum_{\kappa=1}^{\infty} D\left(\frac{(-1)^{\kappa-1}}{\kappa} \Delta^{\kappa} G_{2}(x)\right)=\sum_{\kappa=1}^{\infty} \frac{(-1)^{\kappa-1}}{\kappa} \Delta^{\kappa} G_{1}(x)=D G_{1}(x)
$$

since $G_{1}(x) \in K_{\alpha}$, from which

$$
S_{2}(x)=G_{1}(x)+c_{2} \text {, or } \Delta G_{2}(x)-G_{1}(x)-R_{2}(x)=c_{2},
$$

where, up to this point, $c_{2}$ is an arbitrary constant.

Taking the limit of both sides, in (3.26), as $x \rightarrow \infty$, and noting that $\lim _{x \rightarrow \infty} R_{2}(x)=0$ (from (3.17) with $m=2$ ), and that,

$$
\lim _{x \rightarrow \infty}\left(\Delta G_{2}(x)-G_{1}(x)\right)=\lim _{\xi \rightarrow \infty}\left(\frac{1}{2 !} g(\xi)\right)=0 \quad(x<\xi<x+1)
$$

by assumption (iii), the constant $c_{2}=0$, and finally

$$
S_{2}(x)=\sum_{\kappa=1}^{\infty} \frac{(-1)^{\kappa-1}}{\kappa} \Delta^{\kappa} G_{2}(x)=G_{1}(x)=D G_{2}(x),
$$

meaning that $G_{2}(x) \in K_{\alpha}$, and the theorem has thus been proved for $m=2$.

Proceeding similarly, one may prove Theorem 12 , for $m=3,4,5, \ldots$.

Using Theorem 12, one may extend the class of functions already belonging to some $K_{\alpha}$, as shown in the following theorems:

Theorem 13. The function $\ln x$ and all its antiderivatives are in $K_{0}$.

Proof. It suffices to apply Theorem 12, to the function

$$
g(x)=\frac{1}{x}, x>0
$$

which is completely monotonic on $(0, \infty)$, belongs to $K_{0}$ (by virtue of Theorem 5, for $p=1$ ), and approaches zero as $x \rightarrow \infty$. 
Theorem 14. If $s$ is any real number, $x^{s} \in K_{0}$.

Proof. If $s<0, x^{s} \in K_{0}$, by virtue of Theorem 5 .

If $s=0$, obviously $x^{s} \in K_{0}$.

If $s$ is any positive integer, $x^{s} \in K_{-\infty}$ (Theorem 1), and therefore belongs to $K_{0}$, as well. Let now $0<s<1$, or equivalently $-1<s-1<0$. The function

$$
g(x)=s x^{s-1} \in K_{0}
$$

by virtue of Theorem 5 , and at the same time satisfies conditions (ii) and (iii) of Theorem 12, therefore according again to Theorem 12, any first antiderivative $G_{1}(x)$ of $g(x)$, also belongs to $K_{0}$. One first antiderivative of $g(x)$, is

$$
G_{1}(x)=x^{s} \in K_{0}, \quad 0<s<1 .
$$

If $1<s<2$, or equivalently $-1<s-2<0$, the function

$$
g(x)=s(s-1) x^{s-2} \in K_{0},
$$

by virtue of Theorem 5, and satisfies conditions (ii) and (iii) of Theorem 12 , therefore any second antiderivative $G_{2}(x)$ of $g(x)$ also belongs to $K_{0}$. One second antiderivative of $g(x)$, is

$$
G_{2}(x)=x^{s} \in K_{0}, \quad 1<s<2 .
$$

The generalization is obvious, establishing thus the validity of Theorem 14 , for all real values of $s$.

Theorem 15. If $g(x), \alpha<x<\infty$, is the Laplace transform of $a$ positive function $P(t), 0 \leq t<\infty$, then $g(x)$ and any $m$-th antiderivative $G_{m}(x)$ of $g(x), m=1,2,3, \ldots$ are in $K_{\alpha}$. 
Proof. Let $g(x), \alpha<x<\infty$, be the Laplace transform of a positive function $P(t), 0 \leq t<\infty$, i.e.,

$$
g(x)=\int_{0}^{\infty} e^{-x t} P(t) d t
$$

The function $g(x)$ is completely monotonic in $\alpha<x<\infty$ (see [13]), and $\lim _{x \rightarrow \infty} g(x)=0$. We can also show that $g(x) \in K_{\alpha}$. Indeed, the $\kappa$-th difference of $g(x)$, is

$$
\Delta^{\kappa} g(x)=(-1)^{\kappa} \int_{0}^{\infty} e^{-x t}\left(1-e^{-t}\right)^{\kappa} P(t) d t, \quad \kappa=1,2,3, \ldots
$$

from which

$$
\sum_{\kappa=1}^{\infty} \frac{(-1)^{\kappa-1}}{\kappa} \Delta^{\kappa} g(x)=(-1) \sum_{\kappa=1}^{\infty} \int_{0}^{\infty} \frac{e^{-x t}\left(1-e^{-t}\right)^{\kappa} P(t)}{\kappa} d t
$$

and since the integrand is nonnegative for all $\kappa$ and $t$, interchange of summation with integration is permissible, by Tonelli's theorem, therefore,

$$
\begin{aligned}
\sum_{\kappa=1}^{\infty} \frac{(-1)^{\kappa-1}}{\kappa} \Delta^{\kappa} g(x) & =(-1) \int_{0}^{\infty} e^{-x t}\left(\sum_{\kappa=1}^{\infty} \frac{\left(1-e^{-t}\right)^{\kappa}}{\kappa}\right) P(t) d t \\
& =(-1) \int_{0}^{\infty} e^{-x t} t P(t) d t=D g(x),
\end{aligned}
$$

meaning that $g(x) \in K_{\alpha}$.

(In obtaining (3.30), use of (1.17) has been made).

Thus, the function $g(x)$, as defined in (3.27), satisfies the conditions (i), (ii) and (iii) of the Theorem 12, therefore, any $m$-th antiderivative $G_{m}(x)$ of $g(x)$, belongs to $K_{\alpha}$, and this completes the proof. 


\section{The Difference Equation $\Delta y(x)=y(x+1)-y(x)=g(x)$}

The difference equation

$$
\Delta y(x)=y(x+1)-y(x)=g(x), \quad g(x) \text { given }
$$

was first studied by Krull, in his pioneer work [11], and subsequently by other researchers (see [3], [12], [8], and [9]).

Under certain conditions, imposed on $g(x)$, the solution $y(x)$ of (4.1), can be expressed as a series of differences of functions, finite or infinite, depending on $g(x)$. This series representation of $y(x)$, usually leads to interesting, and often unexpected, applications and results.

To be more specific, let $G(x)$ be an antiderivative of $g(x)$. Let us further assume that $G(x)$ belongs to some $K_{\alpha}$, i.e.,

$$
D G(x)=\sum_{\kappa=1}^{\infty} \frac{(-1)^{\kappa-1}}{\kappa} \Delta^{\kappa} G(x), \quad \alpha<x<\infty .
$$

Equation (4.1) becomes

$$
\Delta y(x)=g(x)=D G(x)=\sum_{\kappa=1}^{\infty} \frac{(-1)^{\kappa-1}}{\kappa} \Delta^{\kappa} G(x), \quad \alpha<x<\infty,
$$

from which one obtains

$$
\begin{aligned}
y(x) & =\sum_{\kappa=1}^{\infty} \frac{(-1)^{\kappa-1}}{\kappa} \Delta^{\kappa-1} G(x) \\
& =G(x)-\frac{1}{2} \Delta G(x)+\frac{1}{3} \Delta^{2} G(x)-\frac{1}{4} \Delta^{3} G(x)+\cdots .
\end{aligned}
$$

Expression (4.3) represents the solution of the difference equation (4.1), in the interval $\alpha<x<\infty$.

Two striking examples of this approach, are given in a recent paper (see [9]), where an expansion for the gamma function $\Gamma(x), x>0$ and the digamma function (or psi function) 


$$
\Psi(x)=\frac{d}{d x}\{\ln \Gamma(x)\}=\frac{\Gamma^{\prime}(x)}{\Gamma(x)}, \quad x>0,
$$

are obtained.

It is well-known that $\Gamma(x)$, satisfies the difference equation

$$
\ln \Gamma(x+1)-\ln \Gamma(x)=\ln x, \quad x>0 .
$$

By Theorem 13, the functions $g(x)=\ln x$ and its antiderivative $G(x)=x \ln x-x$, belong to $K_{0}$, therefore $\ln \Gamma(x)$ admits a series representation according to (4.3). Pursuing this approach to the end, one arrives at the following expression for $\Gamma(x)$, (Equation (3.18) in [9])

$$
\Gamma(x)=\sqrt{2 \pi e} e^{-x} \prod_{\kappa=0}^{\infty}\left[\prod_{j=0}^{\kappa}(x+j)^{(x+j)}\left({ }_{j}^{\kappa}\right)(-1)^{j}\right]^{\frac{1}{\kappa+1}},
$$

or

$$
\Gamma(x)=\sqrt{2 \pi e} e^{-x} x^{x}\left[\frac{x^{x}}{(x+1)^{x+1}}\right]^{\frac{1}{2}} \cdot\left[\frac{x^{x}(x+2)^{x+2}}{(x+1)^{2(x+1)}}\right]^{\frac{1}{3}} \cdot\left[\frac{x^{x}(x+2)^{3(x+2)}}{(x+1)^{3(x+1)}(x+3)^{x+3}}\right]^{\frac{1}{4}} \ldots
$$

Similarly, the digamma function $\Psi(x)$, satisfies

$$
\Psi(x+1)-\Psi(x)=\frac{1}{x}, \quad x>0
$$

Again by Theorems 5 and 13, the function $g(x)=\frac{1}{x}$ and its antiderivative $G(x)=\ln x$, belong to $K_{0}$.

Following the procedure outlined above, one may obtain the following representation for $\Psi(x)$, (Equation (3.22) in [9]):

$$
\Psi(x)=\sum_{\kappa=0}^{\infty} \frac{1}{\kappa+1} \sum_{j=0}^{\kappa}(-1)^{j}\left({ }_{j}^{\kappa}\right) \ln (x+j), \quad x>0 .
$$

In a new paper to appear soon, there will be more interesting applications and results, following the Theory developed in this work. 


\section{References}

[1] H. Alzer and C. Berg, Some classes of completely monotonic functions, Ann. Acad. Scient. Fennicae 27 (2002), 445-460.

[2] C. Berg and H. L. Pederson, A completely monotone function related to the gamma function, J. Comp, Appl. Math. 133 (2001), 219-230.

[3] J. Dufresnoy and Ch. Pisot, Sur la relation fonctionnelle $f(x+1)-f(x)=\Phi(x)$, Bull. Soc. Math. Belgique 15 (1963), 259-270.

[4] W. Feller, Completely monotone functions and sequences, Duke Math. J. 5 (1939), 661-674.

[5] H. van Haeringen, Completely monotonic and related functions, J. Math. Anal. Appl. 204 (1996), 389-408.

[6] M. E. H. Ismail, Integral representations and complete monotonicity of various quotients of Bessel functions, Canad. J. Math. 29 (1977), 1198-1207.

[7] M. E. H. Ismail, Complete monotonicity of modified Bessel functions, Proc. Amer. Math. Soc. 108 (1990), 353-361.

[8] D. P. Kanoussis and V. G. Papanicolaou, The $R$-transform of a real valued function and some of its applications, Journal of Applied Functional Analysis 8 (2013).

[9] D. P. Kanoussis and V. G. Papanicolaou, On the inverse of the Taylor operator, Scientia, Series A: Mathematical Sciences 24 (2013).

[10] D. P. Kanoussis and V. G. Papanicolaou, New expansions of two trigonometric functions, Journal of Concrete and Applied Mathematics 12(3-4) (2014), 331.

[11] W. Krull, Bemerkungen zur Differezengleichung $g(x+1)-g(x)=\Phi(x)$, Math. Nachr. 1 (1948), 365-376.

[12] M. Kuczma, O Rownaniu Funkcyjnym $g(x+1)-g(x)=\Phi(x)$, Zeszyty Naukowe Univ. Jagiell., Mat.- Fiz.- Chem. 4 (1958), 27-38.

[13] K. S. Miller and S. G. Samco, Completely monotonic functions, Integr. Transf. and Special Functions 12(4) (2001), 389-402.

[14] D. V. Widder, The Laplace Transform, Princeton Univ. Press, Princeton, N.J., 1941. 